\title{
Ocular Manifestations in Human Immunodeficiency Virus Patients in the Pre-HAART and HAART Era
}

\author{
Anjana Somanath ${ }^{1, *}$, Rathinam Sivakumar ${ }^{2}$, Radhika Thundikandy $^{1}$, Vedhanayaki Rajesh ${ }^{1}$ \\ ${ }^{1}$ Uvea Consultant, Department of Uvea, Aravind Eye Care System, Madurai, India \\ ${ }^{2}$ Head of Department - Uvea, Aravind Eye Care System, Madurai, India
}

Email address:

anjana471306@gmail.com (A. Somanath)

${ }^{*}$ Corresponding author

\section{To cite this article:}

Anjana Somanath, Rathinam Sivakumar, Radhika Thundikandy, Vedhanayaki Rajesh. Ocular Manifestations in Human Immunodeficiency Virus Patients in the Pre-HAART and HAART Era. International Journal of HIV/AIDS Prevention, Education and Behavioural Science. Vol. 6, No. 1, 2020, pp. 10-14. doi: 10.11648/j.ijhpebs.20200601.12

Received: April 5, 2020; Accepted: April 27, 2020; Published: May 28, 2020

\begin{abstract}
The human immunodeficiency virus infection causes a wide range of ocular manifestations. It is a growing concern in the developing countries. Now, the government provides the highly active antiretroviral therapy free of cost. But ocular manifestations continue to arise in patients with Human Immunodeficiency Virus infection. Hence a comparitive analysis of the disease pattern is important in assessing the ocular manifestations in the pre-HAART and HAART era. The aim of this retrospective series is to analyse the ocular manifestations in Human Immunodeficiency Virus patients in the preHAART and HAART era This retrospective study includes Human Immunodeficiency Virus positive patients from the preHighly Active Antiretroviral Therapy (HAART) (1995-2000) and HAART era (2005-2017). Patients were assessed for the ocular manifestations. This study included 83 patients in the pre HAART era and 98 patients in the HAART era. Cytomegalovirus retinitis was the most common ocular manifestation in the pre-HAART and the HAART era. Inspite of free availability of the antiretroviral therapy, the results of our study has not shown a reduction in the ocular manifestations in the HAART era. Awareness among the high risk population, screening of population at community health centers, private health sectors and engaging non-government organizations for awareness in the community can help in early diagnosis of HIV. This may cause a reduction in the ocular manifestations in individuals with Human Immunodeficiency Virus.
\end{abstract}

Keywords: Cytomegalovirus Retinitis, Human Immunodeficiency Virus, Highly Active Antiretroviral Therapy

\section{Introduction}

Highly Active Antiretroviral Therapy (HAART) has lead to the reduction of ocular diseases and opportunistic infections due to Human Immunodeficiency Virus $[1,2]$. According to the UNAIDS, in 2017 there are 2,100,000 individuals in India are living with Human Immunodeficiency Virus (HIV) and the HIV incidence is 0.1 per 1000 population. Approximately 69,000 deaths are seen due to Acquired Human Immunodeficiency Syndrome (AIDS) [3]. In India, $56 \%$ of individuals living with HIV are on treatment [3]. A significant rise has been seen from 2013, where $36 \%$ of individuals living with HIV received treatment [10].

In the pre-HAART era, HAART therapy was not accessible to all due to the high cost of the drugs [4]. But in the year 2000, due to the production of antiretroviral drugs by Indian generic manufacturers, the cost of the drugs has been reduced. It is accessible to all individuals living with Human Immunodeficiency Virus [4].

The World Health Organisation's (WHO) antiretroviral therapy guidelines for a patient living with HIV under the national program has been revised. As per the revised guidelines, it has been decided to treat all HIV positive patients with antiretroviral therapy regardless of CD4 count, clinical stage, age or population [3].

Ocular manifestations such as cytomegalovirus retinitis, HIV retinopathy, ocular syphilis, ocular tuberculosis, ocular toxoplasmosis, herpes zoster ophthalmicus and acute retinal necrosis can be seen in patients with $\operatorname{HIV}[5,9]$. With the introduction of HAART, CD4 counts increase and decreases the viral load of HIV in an individual with AIDS $[6,7]$. Thus 
with the introduction of HAART therapy there has been a reduction in the ocular manifestations in individuals with HIV [8].

In this retrospective study we have compared ocular manifestations of individuals with HIV in the pre-HAART and HAART era and the effect of antiretroviral therapy on their ocular manifestations.

\section{Methods}

This is a retrospective study done in a tertiary eye care center in Southern India. It includes patients in the preHAART era from 1995 to 2000. In the HAART era it includes patients from 2005 to 2017. All patients were diagnosed cases of HIV or detected to be HIV positive while evaluating them for their ocular manifestations. They have presented to our hospital due to their ocular complaints or referred from an ophthalmologist or treating physician at Antiretroviral Therapy (ART) center for their ocular complaints.

The case records and electronic medical records of patients were assessed for the ocular manifestations and duration of ART. An institutional review board approval was taken for this retrospective study.

The patients have undergone visual acuity testing, slit lamp examination and fundus evaluation. The prevalence of ocular diseases, visual impairment, underlying systemic diseases other than HIV was also noted.

In the HAART era, cytomegalovirus retinitis/acute retinal necrosis/progressive outer retinal necrosis were treated with intravitreal ganciclovir if the lesion was involving or near macula. Intravitreal ganciclovir injection was repeated after 1 week if lesion was still persisting or resolving. Oral valacyclovir $1000 \mathrm{mg}$ three times a day was given irrespective of the CD4 count. Oral valacyclovir was stopped after the CD4 counts improved and lesions had resolved.

Retinal detachment secondary to cytomegalovirus retinitis or HIV positive patient with vitreous hemorrhage was referred to our retina outpatient department in our hospital for further management.

Patients with associated systemic co-morbitidities like tuberculosis were referred to the Antiretroviral Therapy (ART) center for evaluation of tuberculosis and starting antituberculosis treatment.

\section{Results}

This retrospective study includes 83 patients in the pre HAART era and 98 patients in the HAART era. In the HAART era, 22 patients were detected to be HIV positive when they were evaluated for their ocular manifestation. 76 patients were aware of their HIV status. They were either referred from ART centers or private ophthalmologists. 52 patients were lost for follow up in the HAART era.

In the pre-HAART and the HAART era males were more affected compared to females. The most common occupation was agriculture workers, drivers or unemployed. Most common mode of acquiring HIV was heterosexual transmission. 2 patients in the pre-HAART era acquired HIV through blood transfusion.

The most common ocular manifestation in the pre-HAART and the HAART era was cytomegalovirus retinitis. The other ocular manifestations are mentioned in the table. (Table 1: Ocular manifestations in the pre-HAART era and HAART era). In the HAART era, ocular manifestations were common in patient with low $\mathrm{CD} 4$ count. (figure 2 Ocular manifestations and CD4 count).

The most common systemic co-morbidities in the preHAART era included tuberculosis (6 patients) and syphilis (2 patients). Whereas in the HAART era the most common systemic co-morbidity was tuberculosis (44 patients). (Figure 3. Systemic co-morbidities in the pre-HAART and HAART era).

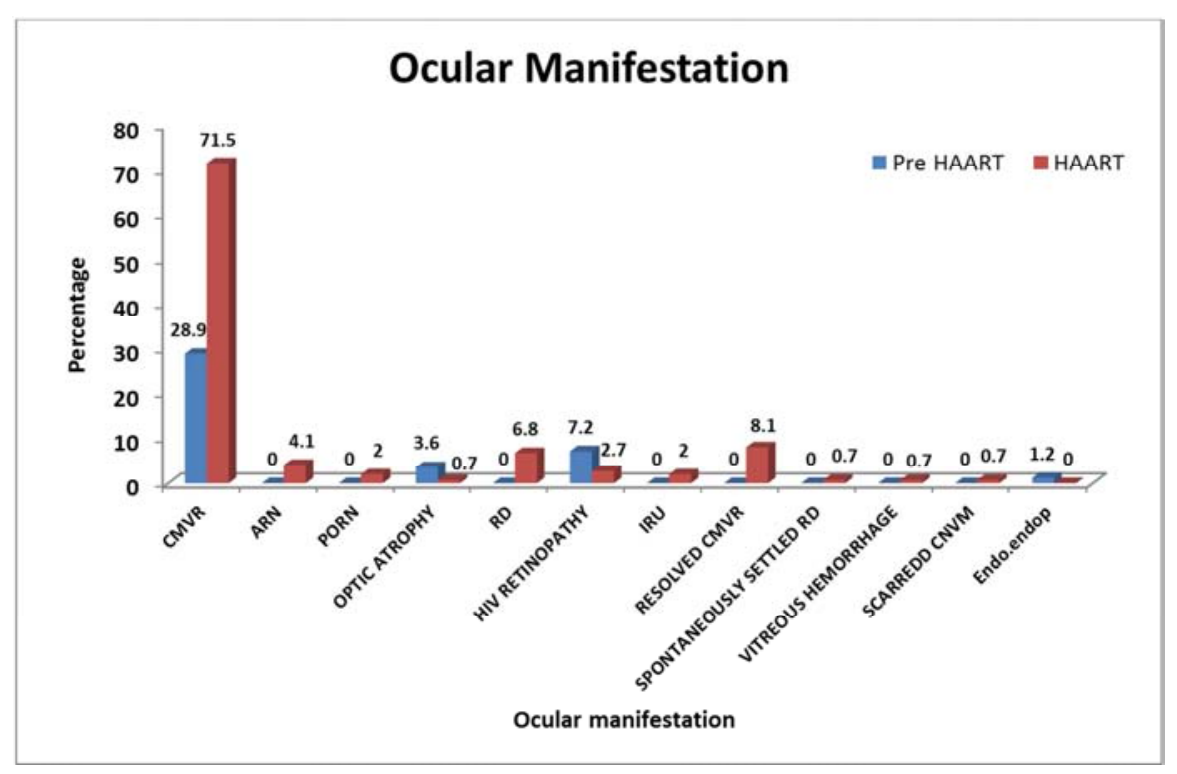

Figure 1. Ocular manifestation in the pre-HAART and the HAART era. 
Table 1. Ocular manifestations in the pre-HAART and HAART era in HIV positive patients.

\begin{tabular}{llll}
\hline SLNO & OCULARMANIFESTATION & PreHAART & HAART \\
\hline 1 & Cytomegalovirus retinitis & 24 & 106 \\
2 & Acute Reinal Necrosis & 10 & 6 \\
3 & Progressive Outer Retinal Necrosis & 1 & 3 \\
4 & Optic atrophy & 1 & 1 \\
5 & Retinal detachment & - & 10 \\
6 & HIV Retinopathy & - & 4 \\
7 & Immune recovery uveitis & - & 3 \\
8 & Resolved cytomegalovirus retinitis & - & 12 \\
9 & Sponaneously settled retinal detachment & - & 1 \\
10 & Vitreous hemorrhage & & 1 \\
11 & Scarred choroidal neovascular membrane & & \\
\hline
\end{tabular}

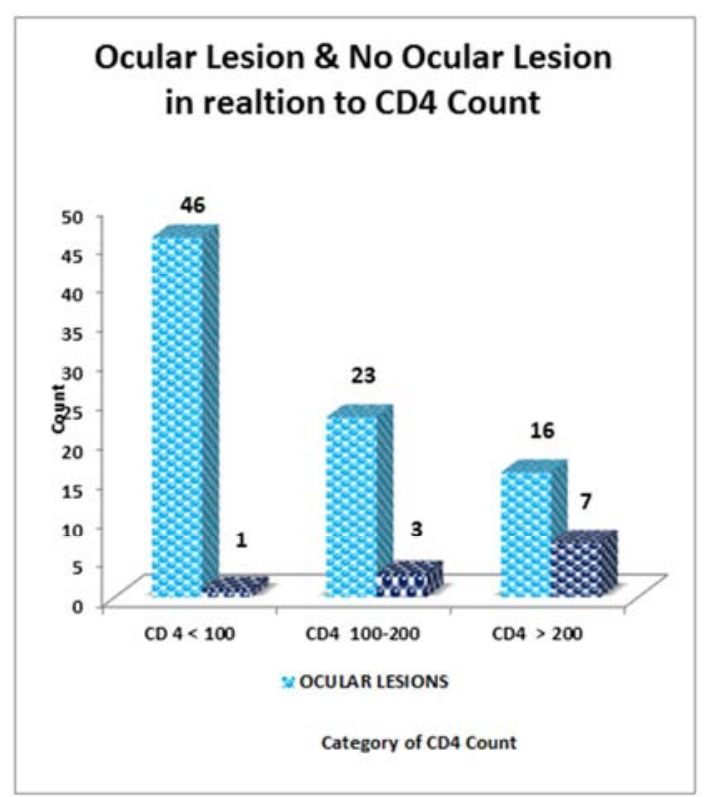

Figure 2. Ocular manifestations and CD 4 count.

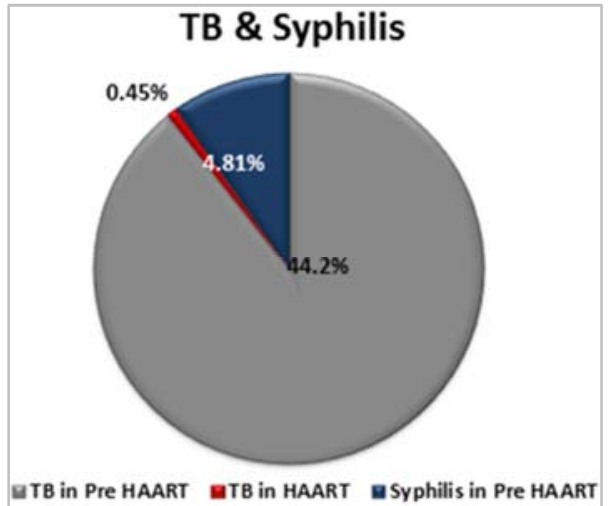

Figure 3. Systemic comorbidities in the pre-HAART and HAART era.

Retinal detachment secondary to cytomegalovirus retinitis was seen in 26 eyes in the HAART era. One eye had spontaneously resolved retinal detachment and one eye had optic atrophy. Immune Recovery Uveitis, a new ocular manifestation due to the advent of HAART was seen in 3 eyes.

Iris tumour was seen in the pre-HAART era. No malignancies were seen in HAART era.
In the HAART era, 44 patients were regular for follow up and were on HAART therapy. 18 patients inspite of being on HAART showed deterioration of vision. Deterioration of vision ranged from no perception of light to visual acuity less than $6 / 36$. The other patients maintained their visual acuity or their vision improved.

\section{Discussion}

In this retrospective study, the ocular manifestations in the pre-HAART and the HAART era continue to remain high inspite of availability of free antiretroviral therapy. This is due to the delayed diagnosis of Human Immunodeficiency Virus and due to discontinuation of antiretroviral therapy.

The national strategic plan 2017-2024 are paving way for an HIV free India by universal coverage of prevention and treatment of individuals with HIV that are effective and equitable [11].

Studies have shown that introduction of HAART has not shown a reduction of ocular manifestations in individuals living with HIV [1]. However, Holland et al have observed that the introduction of HAART has markedly reduced the incidence of cytomegalovirus retinitis but has not eliminated the new cases. Nasodi et al and Accorinti et al have observed a reduction in ocular complications and opportunistic infections with the advent of HAART $[12,13]$.

There has been a steady decline in the prevalence of HIV in India. According to the India HIV 2017 estimation report the prevalence of $\mathrm{HIV}$ is more common in males $(0.25 \%)$ compared to the females $(0.19 \%)[1,14,16$.$] Sex workers,$ homosexuals, drug abusers and the transgenders are the high risk population in India. $[15,17]$ Decrease in the prevalence in the female population might be due to the lack of awareness, social stigma and neglect of the disease. Similarly, in our study females who presented with ocular manifestation in our clinic were aware of the disease. They had not availed the antiretroviral therapy due to family issues or due to social stigma.

Occupation of the individual has also been the cause for the prevalence of HIV. Sharma R and Shukla Y et al have shown that drivers, unemployment and labour workers are the factors responsible for prevalence of $\operatorname{HIV}[16,17]$. Patients seen in our study were agricultural workers, drivers or were unemployed. Unemployment leads to poor income 
leading to one of the important factors for the prevalence of HIV.

Cytomegalovirus retinitis has been the most common ocular manifestation in our study in the pre-HAART and HAART era. This has been the most common ocular manifestation in an individual with HIV in other countries including India $[1,18-20]$. CMV retinitis with the involvement of macula will be one of the most important causes of vision loss. Complications secondary to CMV retinitis like retinal detachment and optic atrophy is seen as in our study. Thorne et al have also concluded that in the HAART era, zone 1 involvement and retinal detachment are the most common cause of vision loss among patients with CMV retinitis [21].

With the advent of HAART, a new ocular manifestation Immune recovery uveitis is seen in HIV individuals. It was seen in $2 \%$ of the patients in our series. Immune recovery uveitis is associated with an increase in CD4 counts [22]. It is characterized by inflammatory complications like macula edema and epiretinal membrane formation [24]. As our patients were lost for follow up, complications secondary to Immune Recovery Uveitis was not seen. Immune recovery uveitis can be treated with anti-inflammatory therapy. Lin et al have reported good response of immune recovery uveitis with anti-inflammatory therapy without reactivation of CMV retinitis [23].

The main limitation of our study is the small sample size. As our hospital is an eye hospital and is not associated with other multispeciality hospital and ART centres, references of individuals with HIV can be missed.

\section{Conclusion}

Inspite of the free availabilty of the antiretroviral therapy in the HAART era, ocular manifestations continue to arise. Awareness among the high risk population, screening of population at community health centres, private health sectors and engaging NGO's for awareness and screening of individuals can help in early diagnosis of HIV and prevent discontinuation of antiretroviral therapy. This may lead to reduction in the ocular manifestations, opportunistic infections and improve the quality of life in individuals with HIV.

\section{Appendix}

Ocular Manifestations in the pre- HAART era. (figures A1 to A4).

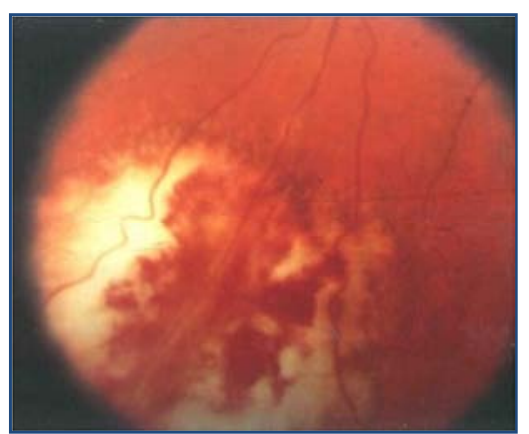

Figure A1. Cytomegalovirus retinitis.

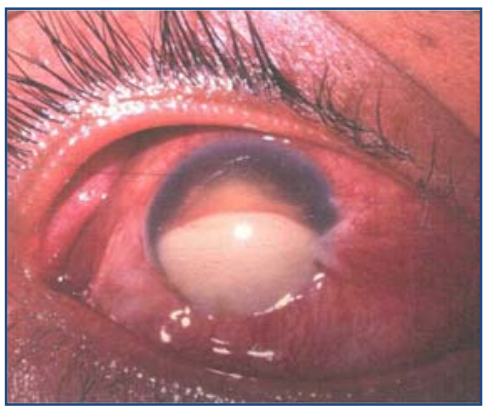

Figure A2. Endogenous endophthalmitis.

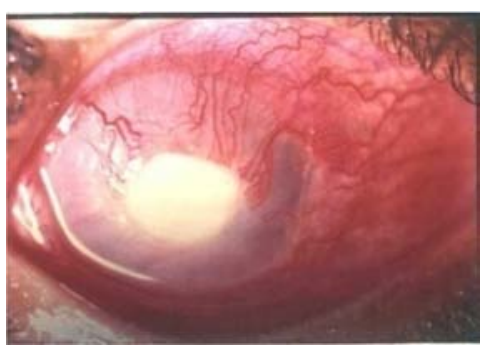

Figure A3. Perforated corneal ulcer:

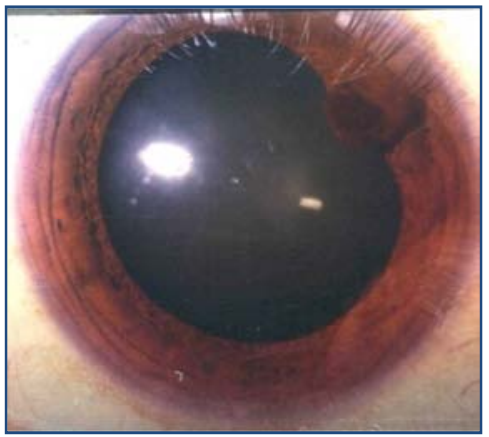

Figure A4. Iris tumour.

Ocular manifestations in the HAART era. (figures A5-A8). 


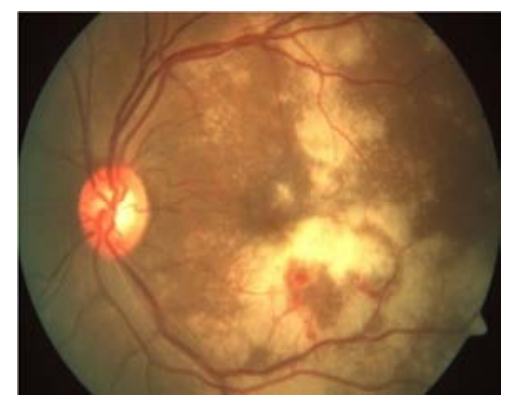

Figure A5. Cytomegalovirus retinitis.

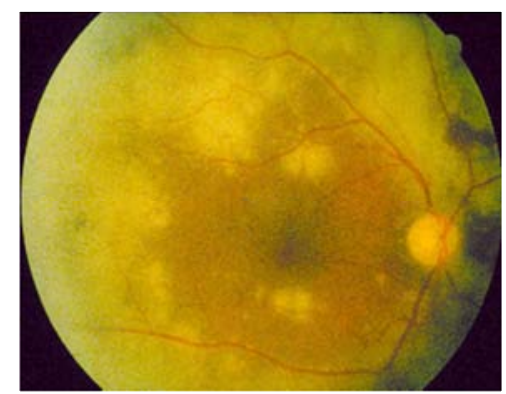

Figure A6. Acute retinal necrosis.

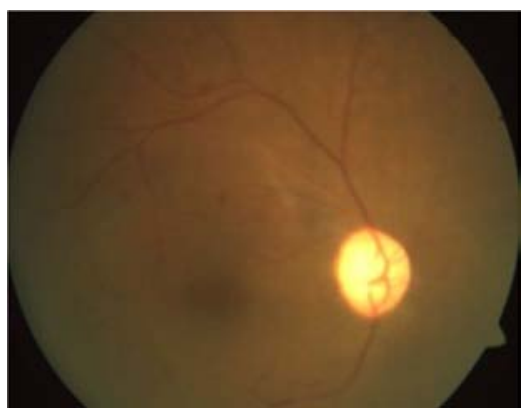

Figure A7. Optic atrophy.

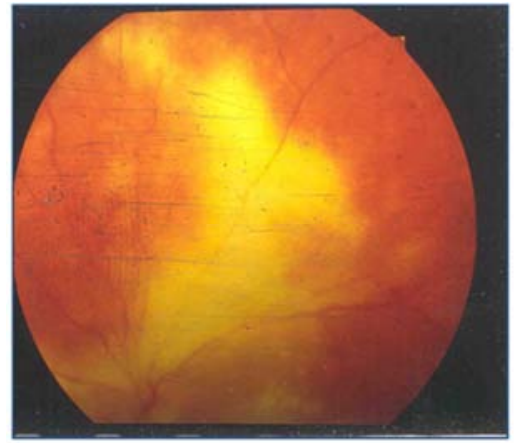

Figure A8. Progressive Outer Retinal Necrosis (PORN).

\section{References}

[1] Aniruddha Agarwal, Ramandeep Singh, Aman Sharmaetal. Ocular Manifestations in Patients with Human Immunodeficiency Virus Infection in the Pre-HAART Versus the HAART Era in the North Indian Population. Ocular Immunology \& Inflammation, 2016; 00 (00): 1-9.

[2] Venkatesh KK, Biswas J, Kumarasamy N. Impact of highly active antiretroviral therapy on ophthalmic manifestations in human immunodeficiency virus/ acquired immunedeficiency syndrome. Indian J Ophthalmol, 2008; 56: 391-3.

[3] UNAIDS data (2018). www.unaids.org. Accessed on 26-52018.

[4] Kumarasamy N, Solomon S, Chaguturu S K, Cecelia AJetal. The changing natural history of HIV disease: before and after the introduction of generic antiretroviral therapy in southern India. Clin Infect Dis. 2005 Nov15; 41 (10): 1525-8.

[5] Sridharan Sudharshan, Sheikh Kaleemunnisha, Akbar Ashraf Banu, Sankaran Shrikrishna, Amala E George, B Rajesh Babu, Bella Devaleenal, Nagalingeshwaran Kumarasamy, Jyotirmay Biswas. Ocular lesions in 1,000 consecutive HIV positive patients in India: along-term study. J Ophthalmic Inflamm Infect. 2013; 3: 2 .

[6] Kumarasamy N, Solomon S, Chaguturu SK, Mahajan A P, Flanigan T P, Balakrishnan P, et al. The safety, tolerability and effectiveness of generic antiretroviral drug regimens for HIV-infected patients in south India. AIDS2003; 17: 226571.

[7] Kumarasamy N, Vallabhaneni S, Flanigan T P, Balakrishnan T P, Cecelia A, Carpenter C J, et al. Rapid viral load suppression following generic highly active antiretroviral therapy in Southern Indian HIV infected patients. AIDS 2005; 19: 625-7.

[8] Venkatesh Kartik K, Biswas J, Kumarasamy N. Impact of highly active antiretroviral therapy on ophthalmic manifestations in human immunodeficiency virus /acquired immunedeficiency syndrome. Indian J Ophthalmol. 2008; 56 (5): 391-393.

[9] Moraes HV, Jr. Ocular manifestations of HIV/AIDS. Curr Opin Ophthalmol. 2002; 13: 397-403.

[10] NACO (2014)'Annual Report2013-14'.

[11] National Strategic plan2017-2024.

[12] Gary N. Holland, AIDS and Ophthalmology: The First Quarter Century. Am J Ophthalmol. 2008 Mar; 145 (3): 397408.

[13] Accorinti M, Pirraglia M P, Corradi R, Corsi C, Fabiani C, Pivetti-Pezzi $\mathrm{P}$. Changing patterns of ocular manifestations in HIV seropositive patients treated with HAART. Eur J Ophthalmol. 2006Sep-Oct; 16 (5): 728-32.

[14] http://naco.gov.in/hiv-facts-figures.

[15] https://www.unaids.org/en/regionscountries/countries/india.

[16] Shukla Y, Rohit B K, Tiwari R, Kasar P K. Sociodemographic profile of people living with HIV/AIDS attending ART center in a tertiary-care hospital in central India. Int J Med Sci Public Health2015; 4: 1464-1467

[17] Sharma R, Profile of HIV-positives and determinants with mode of transmission of HIV /AIDS patients on anti-retroviral treatment center at civil hospital, Ahmedabad. Indian J Sex Transm Dis32: 14-18.

[18] Sudharshan et al. Ocular lesions in 1000 consecutive HIV patients in India: along term study. J ournal of Opthalmic Inflammation and Infection, 2013, 3: 2.

[19] Jabs D A. Ocular manifestations of HIV infection. Trans Am Ophthalmol Soc. 1995; 93: 623-83. 
[20] Biswas J, Madhavan H N, George A E, Kumarasamy N, Solomon S. Ocular lesions associated with HIV infection in India: a series of 100 consecutive patients evaluated at a referral center. Am J Ophthalmol. 2000 Jan; 129 (1): 9-15.

[21] Thorne J E et al. Causes of visual acuity loss among patients with AIDS and cytomegalovirus retinitis in the era of highly active antiretroviral therapy. Ophthalmology. 2006 Aug; 113 (8): 1441-5.

[22] Goldberg D E, Smithen L M, Angelilli A, Freeman W R. HIVassociated retinopathy in the HAART era. Retina. $2005 \mathrm{Jul}-$ Aug; 25 (5): 633-49.
[23] Lin Y C, Yang C H, Lin C P, et al. Cytomegalovirus retinitis and immune recovery uveitis in AIDS patients treated with highly active antiretroviral therapy in Taiwanese. Ocular Immunology and Inflammation. 2008; 16 (3): 83-87.

[24] Karavellas, Marietta $P$ et al. Long-term posterior and anterior segment complications of immune recovery uveitis associated with cytomegalovirus retinitis. American Journal of Ophthalmology, Volume130, Issue1, 57-64. 